\title{
Comparative analysis of distributed mass micromachined gyroscopes fabricated in SCS-SOI and EFAB
}

\author{
Alexander Trusov ${ }^{a^{*}}$, Cenk $_{\text {Acar }}{ }^{b}$ and Andrei M. Shkel ${ }^{a}$ \\ ${ }^{a}$ Department of Mechanical and Aerospace Engineering, Microsystems Laboratory, University of \\ California Irvine, Engineering Gateway 2110, Irvine, CA 92697, USA \\ ${ }^{b}$ BEI Systron Donner Automotive Division, 2700 Systron Drive Concord, CA 94518-1399,USA
}

\begin{abstract}
This paper studies a design approach that yields robust vibratory MEMS gyroscopes. The design is based on multiple drive-mode resonators with incrementally spaced frequencies, distributed symmetrically around the center of a supporting frame. These resonators are structurally constrained in the tangential direction with respect to the supporting frame. In the presence of an angular rotation rate about the z-axis, a harmonic Coriolis force is induced on each proof mass. These force vectors lie in the tangential direction, generating a resultant torque on the supporting frame. The net Coriolis torque excites the supporting frame into torsional oscillation about the z-axis, which is capacitively detected to generate angular rate measurement. Two batches of prototypes have been fabricated using in-house single crystal silicon on insulator (SCS-SOI) bulk-micromachining and $\mathrm{EFAB}^{\mathrm{TM}}$ process commercially available from Microfabrica. Wideband drive operation was demonstrated in SOI devices. EFAB process yielded $850 \mathrm{~Hz}$ devices with quality factor 250 in air (bandwidth $3 \mathrm{~Hz}$ ) and 850 in vacuum. Increase of temperature from $25^{\circ}$ to $125^{\circ} \mathrm{C}$ shifts the resonant frequency down by roughly bandwidth, while quality factor drops by $8 \%$. Parasitics model associated with EFAB consists of only a lumped capacitor and is simpler than two-parametric parasitics circuit in SOI devices. Nonlinear parametric excitation of motion at resonant frequency by super-harmonic AC voltage was experimentally characterized. This actuation method provides high amplitude of motion and separates motion from parasitics in frequency domain. The actuation method can potentially further improve the bandwidth and gain characteristics of distributed mass gyroscope.
\end{abstract}

Keywords: MEMS, Gyroscope, wide-bandwidth, EFAB, SOI

\section{INTRODUCTION}

Conventional gyroscope designs based on matching (or near-matching) the drive and sense modes are extremely sensitive to variations in oscillatory system parameters that shift the natural frequencies and introduce quadrature errors. Non-conventional design concepts have been reported that increase bandwidth to improve robustness, but with the expense of response gain reduction. This paper presents an experimental evaluation of the approach that can yield robust vibratory MEMS gyroscopes with better gain characteristics while retaining the wide bandwidth. The approach was originally presented in Ref.1 with some preliminary characterization in Ref. 2. It is based on forming multiple drivemode oscillators with incrementally spaced frequencies, distributed symmetrically around the center of a supporting frame (see Fig.1). Oscillators are driven in-phase towards the center of symmetry, and are structurally constrained in the tangential direction with respect to the supporting frame. When the structure is subjected to z-axis angular rate, a harmonic Coriolis force induced on each proof mass, combining to generate a resultant torque on the supporting frame. The net Coriolis torque excites the supporting frame into torsional oscillation about the z-axis, which is detected capacitively.

Two batches of prototypes have been fabricated using in-house single crystal silicon on insulator (SCS-SOI) bulkmicromachining and $\mathrm{EFAB}^{\mathrm{TM}}$ process (commercially available from Microfabrica). The SOI fabrication process relies on deep-reactive ion etching (DRIE) through device layer of a silicon-on-insulator wafer and front-side release of the structures by etching the sacrificial Oxide layer in HF solution. EFAB ${ }^{\mathrm{TM}}$ process consists of number of sequential electro-depositions and planarizations of patterned metal structural layers on a substrate. System identification was performed for the fabricated prototypes. Lumped parasitic model for SOI process was modeled and identified in terms of

*atrusov@uci.edu; phone 1949 824-6314; fax 1949 824-8585; http://mems.eng.uci.edu/

Smart Structures and Materials 2006: Sensors and Smart Structures Technologies for Civil,

Mechanical, and Aerospace Systems, edited by Masayoshi Tomizuka, et al., Proc. of SPIE Vol. 6174, 61742A, (2006) -0277-786X/06/\$15 - doi: 10.1117/12.658932

Proc. of SPIE Vol. 6174 61742A-1 
lumped parasitics capacitance and substrate resistance. The parasitics model for EFAB is one-parametric and consists of lumped stray capacitance in parallel to the ideal system. In both cases parasitics was identified and eliminated by postprocessing of the experimentally obtained frequency responses. Also, an inherently parasitics free method of frequency response acquisition was developed using EAM procedure. Parasitic free frequency responses were used to extract structural parameters of the prototypes. The main mode of EFAB prototypes has natural frequency of $890 \mathrm{~Hz}$, with quality factor 250 in air and 850 in 30 mTorr vacuum. Experimental investigation of the effect of temperature on the structural parameters of the prototypes showed that the resonant frequency drops with temperature. Increase of temperature from $25{ }^{\circ} \mathrm{C}$ to $125^{\circ} \mathrm{C}$ resulted in $\sim 0.5 \%$ resonant frequency reduction and $\sim 8 \%$ decrease in quality factor.

Either lateral comb or parallel plate configuration of drive capacitor can be used to actuate the gyroscope. Usually lateral combs are preferred because they generate displacement-independent force. Parallel plate drive capacitors, however, generate force which is a highly nonlinear function of the displacement. We study application of parallel plate capacitor nonlinearity to drive the distributed mass gyroscope into oscillation at resonance frequency by applying superharmonic AC voltage. Using EFAB prototypes we experimentally demonstrate high gain of this nonlinear parametric resonance, which also allows for motional amplitudes above $80 \%$ of the gap without snapping. Additionally, this actuation method provides frequency domain separation between motion and parasitics, making the motional signal parasitics free. Recently we designed a new set of prototypes, including devices optimized for parametric excitation. These prototypes will be fabricated in SCS-SOI and SOG processes.

\section{DESIGN CONCEPT}

The studied design concept is presented in Ref.3 and is aimed to provide improved bandwidth and gain characteristics by utilizing a number of drive mode resonators. These drive mode resonators are distributed symmetrically around the center of a supporting frame. They have equally spaced natural frequencies and are driven in-phase towards the center of symmetry. Resonators are structurally constrained in the tangential direction with respect to the supporting frame. Each of them is driven at the same drive frequency. In the presence of an angular rotation rate about the z-axis, a harmonic Coriolis force at the drive frequency is induced on each proof mass in the direction orthogonal to each drive-mode oscillation directions. Thus, each of the induced Coriolis force vectors lies in the tangential direction, combining to generate a resultant torque on the supporting frame. The net Coriolis torque excites the supporting frame into torsional oscillations about the z-axis, which are detected by sense capacitors for angular rate measurement.

The Coriolis force acting on a moving body is proportional to its velocity. Thus, for resonator with fixed motional frequency both the Coriolis force and the sense-mode response are proportional to the drive-mode oscillation amplitude. The drive-mode amplitude can be increased by increasing the $Q$ factor with vacuum packaging and operating at the peak of the drive-mode frequency response. However, large drive-mode amplitude and bandwidth can not be achieved at the same time in a 1-DOF drive system. The investigated approach explores the possibility of increasing the drive-mode response bandwidth of micromachined gyroscopes by utilizing multiple resonators with incrementally spaced resonant frequencies in the drive-mode. The drive and sense modes are effectively decoupled by forming a multi-directional linear drive-mode that transmits the Coriolis force into a torsional sense-mode.

In the distributed mass gyroscope with the multiple drive-mode oscillators wide-bandwidth drive mode response is achieved, leading to improved robustness to structural and thermal parameter fluctuations. Enhanced mode-decoupling is accomplished by distributing the linear drive-mode oscillators radially and symmetrically, to form a multi-directional linear drive-mode and a torsional sense-mode; minimizing quadrature error and zero-rate-output. A wide-bandwidth operation region is achieved in the drive-mode frequency response, by designing or actively tuning the resonance frequency of each drive-mode oscillator to be incrementally spaced. Since the tangential Coriolis forces induced on each proof mass jointly generate a resultant torque on the supporting frame, a "levelled" total Coriolis torque is achieved over a wide range of driving frequencies. The device is nominally operated in this levelled region of the Coriolis torque frequency response, so that fluctuations in system parameters that shift oscillator resonance frequencies will not result in a significant change in the total Coriolis torque. If the sense-mode resonance frequency is designed to be accommodated in the same frequency band, the requirement on the degree of mode-matching is relaxed, and robustness against structural and thermal parameter fluctuations is achieved. 


\section{ACTUATION, SENSING, AND PARASITICS}

In this section we discuss principles of capacitive actuation and sensing in distributed mass gyroscope and associated parasitics. Methods and experimental setups for electrostatic characterization of prototypes are developed.

Similarly to other types of vibratory gyroscopes, distributed mass gyroscope relies on drive mode oscillation to create a unit sensitive to Coriolis force. In the area of capacitive vibratory MEMS gyroscopes a combination of DC and AC voltages is often used to create time varying attractive force in the drive capacitor. In this configuration motional and parasitic signals have power concentration at the same frequencies. The Coriolis force sense signal occurs at the frequency of motion and is corrupted by the feed-through parasitics.

Let us consider the electromechanical model of the gyroscope's arbitrary vibrational mode (e.g. drive or sense) characterization shown in Fig. 2. The moving mass of resonator is represented by a solid square. It is suspended relative to the fixed structural anchors with a spring-dashpot system. Fixed drive and sense electrodes surround the moving mass on the two sides. The anchored electrode of the drive capacitor is shown by a parallel plate fixed on the left side of the moving mass. The AC driving voltage $V_{d}(t)$ is applied to this electrode. Anchored electrode of the sense capacitor is shown by a fixed plate parallel to the body of resonator on the right. It is virtually grounded using a trans-impedance amplifier. The output current $I(t)$ is converted to the voltage $V(t)$ with gain $-R$. The output of the amplifier is connected to signal processing hardware, such as HP35665A dynamic signal analyzer.

It is important to notice that pair of anchored drive and sense electrodes also forms a capacitor (see Fig. 3). The AC driving voltage across this capacitor results in a flow of parasitics current. Together with other forms of electrical coupling (such as conductance through the substrate) capacitive coupling is responsible for feed-through of the applied voltages into the sense output. The feed-through effects can be modeled by linear circuit that electrically couples anchored sense electrodes directly to anchored drive electrodes, as well as other parts of the device. Different levels of circuit complexity and structure can be adequate for devices fabricated in different processes. However, the main feature of the linear circuit to respond at excitation frequencies remains common. The parasitics feed-through occurs only at the frequencies of the applied voltages. If the parasitics is purely capacitive, then only the AC voltages result in feed-though. When applied $\mathrm{AC}$ voltage is a perfect harmonic wave feed-through has one frequency component. As we demonstrated in this work, parasitics in gyroscopes fabricated with EFAB process can be described by a single stray capacitor $\left(C_{p}\right)$, while for the SOI devices at least two-parametric parasitics model is needed (capacitance $C_{p}$ and substrate resistance $R_{p}$ ).

The mechanical part of capacitive MEMS resonator is considered to be a lightly damped mass-spring system that acts as a linear low pass filter of the force applied. When the potential difference in drive capacitor is sum of voltages $V_{d c}=$ const and $V_{d}(t)=v_{d} * \sin \left(w_{d}{ }^{*} t\right)$ the body of resonator is mainly excited by $V_{d c} * V_{d}(t)$ proportional forcing term (assuming $V_{d c}>>v_{d}$ ). The motion mostly occurs at the drive AC voltage frequency $w_{d}$. Virtually grounded sense electrode capacitively picks up both motional current (caused by change in sense capacitance $C_{s}(t)$ during the motion) and feedthrough (caused by excitation of parasitics circuit with AC drive voltage). In this configuration both motional and parasitic signals occur at the same frequencies and motional signal is corrupted by the feed-through. The studied vibratory mode of the gyroscope is assumed to consist of linear single degree of freedom resonator with mass $m$, natural frequency $w_{n}$, and quality factor $Q$. According to the laws of electrostatics, the equation of motion for resonators displacement $x(t)$ is given by

$$
\ddot{x}(t)+\frac{\omega_{n}}{Q} \dot{x}(t)+\omega_{n}{ }^{2} x(t)=\frac{1}{2 m} \frac{\partial C_{d}(x)}{\partial x} V^{2}(t),
$$

where the potential difference across the drive capacitor is given by $V(t)=V_{d c}+V_{d}(t)=V_{d c}+v_{a c} \sin \left(\omega_{d} t\right)$ and $C_{d}(x)$ is drive capacitance as function of displacement. The right hand side of the equation can be written as a sum of three forcing terms, applied simultaneously at $0, w_{d}$, and $2 w_{d}$ frequencies:

$$
\ddot{x}(t)+\frac{\omega_{n}}{Q} \dot{x}(t)+\omega_{n}{ }^{2} x(t)=\frac{1}{2 m} \frac{\partial C}{\partial x}\left\{\left(V_{d c}^{2}+\frac{1}{2} v_{a c}^{2}\right)+\left(2 V_{d c} v_{a c} \sin \left(\omega_{d} t\right)\right)-\left(\frac{1}{2} v_{a c}^{2} \cos \left(2 \omega_{d} t\right)\right)\right\} .
$$


Let us assume the drive capacitor is formed by lateral combs. Then $\partial C(x) / \partial x=C_{x}=$ const and the equation describes a one degree of freedom linear time invariant (LTI) system with inputs at three different frequencies. Commonly used driving scheme is $w_{d} \sim w_{n}$. In this case $2 w_{d}$ lies outside of the mechanical bandwidth of the resonator and the motion is a sum of steady state displacement and harmonic oscillation at the drive frequency $w_{d}$. The main harmonics forcing term is given by $1 / m C_{x} V_{d c} v_{a c} \sin \left(\omega_{d} t\right)$.

Alternatively, drive AC voltage frequency can be selected to be half of the vibratory mode natural frequency (i.e. $w_{d} \sim$ $\left.0.5 w_{n}\right)$. In this case $-1 / m C_{x} v^{2}{ }_{a c} \cos (2 \omega t)$ harmonic term on the right hand side becomes predominant.

In the scope of LTI system approach these are the main choices of gyroscope's driving scheme. The first one provides more forcing amplitude at smaller values of AC voltage and causes less capacitive parasitics for the same amount of motion. The same DC bias can also be used for sensing of the drive motion. On the negative side, both motion and parasitics occur at the same frequencies and thus cannot be separated by means of band-pass filtering.

The second scheme uses only $\mathrm{AC}$ voltage and provides frequency separation between applied $\mathrm{AC}$ voltage and motion. However, it requires relatively large $\mathrm{AC}$ voltage amplitudes to actuate the motion, which decreases overall signal to noise ration (SNR). In practice most sources of AC voltage provide some undesired amount of energy at harmonics of the main wave frequency. This means that parasitics feed-through of the second harmonic can overlap with motional signal, occurring at $2 w_{d} \sim w_{n}$, and corrupt it.

We can see that it can be very advantageous to drive (i.e. provide AC voltage) at frequency, higher than the motional natural frequency. Luckily, this can be done using nonlinearity of parallel plates to create parametric excitation. The experimental characterization of this nonlinear approach is described later in this paper.

\section{PROTOTYPES FABRICATED IN SCS-SOI PROCESS}

In this section we summarize characterization results on silicon prototypes presented in Ref. 3. The wide-bandwidth design concept was analyzed experimentally on the bulk-micromachined prototype structures, fabricated in the UCI Integrated Nano-Systems Research Facility (INRF). A one-mask process based on SOI (Silicon-on-Insulator) wafers was developed and optimized for high-aspect ratio structures. The process relies on deep-reactive ion etching (DRIE) through the 100 micrometer thick device layer, and front-side release of the structures by etching the Oxide layer in HF solution. In the device, 15 micrometer wide holes were used to perforate the suspended structures, and 10 micrometer gaps were used in the sensing and actuation capacitors. The anchors were designed as solid areas large enough to perform wirebonding. The natural frequencies of identically designed drive mode resonators are slightly different due to fabrication imperfections.

The dynamic response of the linear drive-mode oscillators and the torsional sense-mode of the prototype gyroscope were characterized in an MMR Vacuum Probe Station. The experimental transfer function was acquired under varying pressure values and at room temperature, using off-chip transimpedance amplifiers with a feedback resistor of $1 \mathrm{MOh}$ connected to an HP Signal Analyzer in sine-sweep mode. The drive-mode frequency responses were acquired utilizing one-port actuation and detection. The driving AC signal plus the DC bias voltage was imposed on the gyroscope structure through the anchor, and the anchored drive electrode was virtually grounded by the transimpedance amplifier. From the electrically acquired frequency response we extract both parameters of the mechanical subsystem and lumped parasitics circuit parameters. The parasitic drive mode parameters are identified to be capacitance between the bonding pads and the substrate $C_{p}=1.157 \mathrm{pF}\left(7.18 \mathrm{pF}\right.$ in case of sense mode) and substrate resistance $R_{p}=1.701 \mathrm{MOh}$.

The resonance frequencies of the drive-mode resonators were observed to be scattered between $4.546 \mathrm{KHz}$ and 5.355 $\mathrm{KHz}$ within an $809 \mathrm{~Hz}$ frequency band. The $16.36 \%$ maximum frequency deviation of the identically-designed drivemode resonance frequencies results purely from the fabrication imperfections. In the presence of this wide-band scatter, measuring the bandwidth of the drive-mode oscillators is crucial to assess the feasibility of the design concept. The bandwidth of the drive-mode response even at atmospheric pressure was observed to be too narrow to achieve wide-band operation without electrostatic tuning of the drive-mode frequencies. Thus, the prototype with the parallel-plate actuated 
drive-mode oscillators which provide a wider range of electrostatic tuning was tested, and the resonance frequency of each oscillator was electrostatically tuned to achieve uniform and smaller frequency separation. After electrostatic tuning of the parallel-plate oscillators for $10 \mathrm{~Hz}$ spacing, the close spacing of the drive-mode resonance frequencies allowed all of the resonators to be excited together, to jointly generate a resultant Coriolis torque. The total Coriolis torque, which is estimated by summing the experimentally measured response of the eight drive-ports, was observed to provide a levelled range of over $90 \mathrm{~Hz}$. When the experiments were repeated at reduced pressures, the resonance peaks in the levelled region of the overall response became more emphasized, as was theoretically illustrated in the previous section. Based on the experimental results, it was concluded that 200 to 300 Torr is the optimal pressure for the parallel-plate devices to achieve a leveled wide-bandwidth drive-mode response with $10 \mathrm{~Hz}$ spacing. When the resonant frequencies were tuned for $5 \mathrm{~Hz}$ spacing, the total drive-mode response gain was measured to be $65 \%$ larger gain, but the bandwidth was observed to drop to $50 \mathrm{~Hz}$. For an additional batch of redesigned devices the resonance frequencies of the drive-mode resonators were observed to be scattered between $6.490 \mathrm{KHz}$ and $6.920 \mathrm{KHz}$ in a $430 \mathrm{~Hz}$ frequency band, with a $6.21 \%$ maximum frequency deviation. The frequency separation of the resonators was observed to provide over $600 \mathrm{~Hz}$ operating frequency region with levelled output in atmospheric pressure. However, the levelled region showed a maximum variation of $17.2 \%$ in the total response due to the non-uniform frequency separation.

\section{PROTOTYPES FABRICATED IN EFAB PROCESS}

The second batch of prototypes was fabricated using EFAB ${ }^{\mathrm{TM}}$ process commercially available from Microfabrica. System identification was performed and showed feasibility of the process for capacitive vibratory MEMS devices.

\subsection{Description of the EFAB Process}

Figure 4 shows SEM micrograph gallery of prototypes, fabricated in EFAB process. The core of EFAB ${ }^{\mathrm{TM}}$ process is a sequential electroforming of multiple structural layers. Substrate of the 5 by 5 millimeter square dies is formed by 0.5 millimeter thick alumina $\left(\mathrm{Al}_{2} \mathrm{O}_{3}\right)$. The first Ni-Co structural layer of 10 micrometer thickness is deposited using electroplating and patterned. Then, blanket $\mathrm{Cu}$ layer is electroformed and planarized to the thickness of the structural layer. The cycle of these two steps is repeated 20 times, after which the sacrificial $\mathrm{Cu}$ layer is chemically etched away. As a result, a 200 micrometer high 3D metal structure on a non-conductive substrate is formed. During the fabrication run the minimal feature was 20-30 micrometers. After the fabrication we package device dies in ceramic DIP packages and wirebond them for the convenience of characterization (see Fig. 5). The used fabrication tolerances did not allow for fabrication of a fully functional gyroscope. However, we characterized several of the vibration modes in the fabricated prototypes and studied effect of pressure and temperature on structural parameters of the prototypes.

\subsection{FEA of the Prototype}

Finite element analysis of the 3D designs was performed using MSC Patran/Nastran software. Torsional vibration mode of the frame was identified to be the lowest frequency mode at about $1 \mathrm{KHz}$. The second identified mode was out-ofplane torsional vibration at about $3.5 \mathrm{KHz}$ (see Fig. 6). Out-of-plane translation with approximately $4 \mathrm{KHz}$ frequency was identified as a third mode. Other modes of vibration in the structure have higher frequencies. The low frequency torsional mode of vibration corresponds to the sense mode of the gyroscope. It was designed to have two separate parallel plate structures, which allow for differential sensing, or, alternatively, two-port actuation and sensing. The mode was experimentally studied. In the following sections we identify the natural frequency of this mode to be $850 \mathrm{~Hz}$. The discrepancy between experimental and FEA values is attributed to the use of relatively coarse mesh.

\subsection{Electrostatic Tuning and Identification of Unbiased Natural Frequency}

In order to successfully operate a capacitive vibratory gyroscope it is important to identify the natural frequencies of operational modes. When parallel plates are used for actuation of particular mode, negative electrostatic spring effect allows for frequency tuning Ref. 4. Natural frequency becomes a decaying function of the applied DC voltage. Typically a significant $\mathrm{DC}$ voltage is used in combination with $\mathrm{AC}$ voltage to create harmonic capacitive force in a parallel plate capacitor. In this case frequency of detectable resonance can be noticeably lower than the true unbiased mechanical natural frequency of the mode. We experimentally measured resonant frequency at different levels of DC voltage applied to the drive parallel plate capacitor by observing the motion of the structure through a microscope. We collected these data-points (resonant frequency versus DC bias) and performed a curve fit, using the linear negative electrostatic spring

Proc. of SPIE Vol. 6174 61742A-5 
analytical model. The resulting curve is shown in Fig. 7 and gives prediction of resonant frequency at different DC bias voltages. Particularly, the value of the fit at zero DC gives the unbiased mechanical frequency of $890 \mathrm{~Hz}$. The resonant frequency of the mode can be tuned without snapping down to $845 \mathrm{~Hz}$ (corresponds to $\sim 120 \mathrm{~V}$ bias).

\subsection{Parasitic Free Acquisition of Frequency Response and System Identification}

Electro-mechanical amplitude modulation (EAM) is a known method of real-time motion sensing in capacitive vibratory microstructures. It allows for frequency domain separation of motional signal from the parasitics feed-through of drive voltages. EAM method consists of applying an AC carrier voltage to the body of the resonator. In combination with physics of capacitive transduction this setup results in an amplitude modulation of the motional signal (i.e. displacement of the resonator). The modulation is a double sideband with non-suppressed carrier type. At the same time the drive voltage feed-through is preserved at its original frequencies.

Demodulation of the EAM pickup signal can yield value of motional amplitude. Double stage demodulation of EAM signals using a lock-in amplifier and a dynamic signal analyzer was used in this work. The signal processing consists of two identical demodulation steps. They are performed first at carrier frequency and then at driving frequency (which the main frequency of motion). A Signal Recovery Model 7265 digital lock-in amplifier performs first stage demodulation of the EAM pick-up signal. This step maps the power from two informational sidebands to one wave at drive frequency. It is important to correctly choose parameters of the fist demodulation stage low pass filter. The pass-band of this filter needs to include the drive frequency $w_{d}$; higher frequencies need to be attenuated. For the second demodulation step we used an HP35665A Dynamic Signal Analyzer, which was used to drive the experiment. This second demodulation stage maps power from drive frequency to zero frequency bias offset. The described dual stage demodulation procedure yields output voltage proportional to the motional amplitude.

The described EAM modulation/demodulation procedure using a lock-in amplifier and signal analyzer can be used to acquire amplitude of parasitic free frequency response of vibration modes in capacitive MEMS device. In our work we set the lock-in amplifier to the carrier frequency of $20 \mathrm{KHz}$. The dynamic signal analyzer was set into sine sweep mode to scan frequencies in the band of interest. During the experiment, amplitude of motion corresponding to each value of drive frequency was registered. Finally, we get a curve of motional amplitude versus drive frequency. Once we obtain the amplitude of the vibratory mode frequency response, we perform a nonlinear least squares fit with appropriate analytical expression, which includes resonant frequency and quality factor as parameters (see Fig. 8). Using this method for the main mode of distributed mass gyroscope prototype fabricated in EFAB process we found that $130 \mathrm{~V}$ biased resonant frequency is $850 \mathrm{~Hz}$ and quality factor in air is 250 (these correspond to mechanical bandwidth of approximately $3.4 \mathrm{~Hz}$ ).

\subsection{Conventional Acquisition of Frequency Response}

Conventional acquisition of frequency response is based on using a single dynamic signal analyzer in a sine sweep mode. The output $\mathrm{AC}$ voltage is combined with a fixed DC voltage to create a potential difference across a drive capacitor and generate the excitation force. The motion is sensed by applying the fixed DC voltage across the sense capacitor. Both drive and sense capacitors are formed by pair of electrodes - one anchored and the other one attached to the moveable body. The AC current produced in the sense capacitor is a sum of velocity proportional sense current and parasitics current, caused by electrical coupling (either pure AC or composite DC/AC) of the fixed sense electrode to the anchored fixed electrode. The experimental transfer function obtained using this setup is a sum of ideal electromechanical transfer function and transfer function of parasitics circuit. If the appropriate model for parasitics is developed, the two parts can be identified separately, yielding both mechanical and parasitics parameters.

We performed the conventional system identification for the prototypes of distributed mass gyroscope. From inspecting real and imaginary parts of the experimentally obtained transfer functions we conclude that the effect of parasitics in this case can be modeled by one lumped capacitor. The contribution of this stray parasitics capacitor is a straight line in the imaginary part of transfer function. Once we identify the inclination of this imaginary line (see Fig. 9), which is this proportional to the stray capacitance in the devices and experimental setup, we eliminate its contribution by subtracting it from the experimentally obtained complex valued transfer function. The result is parasitic free complex transfer function of the vibrational mode under test (see Fig. 10). This system identification procedure was used to characterize EFAB distributed mass prototypes in open air and vacuum, as well as at different temperatures. Lumped 
parasitics capacitance in the sense mode of EFAB distributed mass prototypes was estimated to be roughly $C_{p}=10 \mathrm{pF}$, which is comparable to similar parameters of SOI parasitics.

\subsubsection{Effect of pressure on bandwidth}

Distributed mass gyroscope prototypes fabricated in EFAB process structurally consist of multiple electroformed layers. We showed that quality factor of devices significantly increases in vacuum and structural damping is not the critical limiting factor at atmospheric pressure. We characterized devices in air and determined $60 \mathrm{~V}$ DC biased resonant frequency of $880 \mathrm{~Hz}$ and quality factor $Q=275$ (corresponds to mechanical bandwidth of $3.2 \mathrm{~Hz}$ ). In $30 \mathrm{mTorr}$ vacuum resonant frequency was detected to be the same, while quality factor increased to $Q=860$ (corresponds to mechanical bandwidth of approximately $1 \mathrm{~Hz}$ )

\subsubsection{Effect of temperature on resonant frequency and quality factor}

The effect of temperature on structural behavior of the metallic EFAB prototypes was also experimentally studied. We mounted the test-bed on a controllable hot plate and acquired experimental frequency responses at 25, 50, 75, 100, and $125{ }^{\circ} \mathrm{C}$. After the post-processing parasitics elimination we extracted the structural parameters for each value of temperature. We concluded that with increase of operational temperature both resonant frequency and quality factor decrease. The raise of temperature from $25^{\circ} \mathrm{C}$ to $125^{\circ} \mathrm{C}$ results in $0.5 \%$ decrease of resonant frequency (equivalent to 4 $\mathrm{Hz}$, which is greater than bandwidth), while the quality factor decreases by approximately $8 \%$. A more rigorous analysis is needed to identify the exact cause and mechanism of these effects.

\section{PARAMETRIC EXCITATION}

As we discussed earlier, it can be very advantageous to drive the gyroscope into oscillatory motion by using a superharmonic frequency $\mathrm{AC}$ voltage because this prevents parasitics at the frequency of motion. In this section we discuss how nonlinearity of parallel plate capacitors can be used to actuate high amplitude motion using nonlinear parametric excitation with a resonance.

\subsection{Analytical Background}

Let us consider a drive mode of a gyroscope, actuated by parallel plate drive capacitor. The major change compared to lateral combs is that capacitance becomes a highly nonlinear function of the motional displacement. The nonlinearity of the drive capacitance results in displacement-dependent actuation force. The dynamic system is a nonlinear system with time varying coefficients. The displacement nonlinear capacitance between drive parallel plates can be written as an infinite power series. Approximations to the full nonlinear dynamics of the drive mode can be derived by considering only a certain number of low power terms in the infinite power expansion. The first order approximation predicts excitation of resonance by a super-harmonic voltage. However, the linear approximation is not sufficient for quantitative analysis of the experimentally observed phenomena.

The drive capacitor $C_{d}(t)$ is formed by a pair of mobile and anchored parallel plate structures. Let us denote media permittivity in the capacitor with $\varepsilon$, initial gap between plates at rest with $g$, individual parallel plate pair overlap length $L$ and plate height (i.e. structural layer thickness) with $y$. The total overlap area in the sense capacitor is given by $A=N^{*} L^{*} y$ where $N$ is a number of parallel plate pairs in the structure. The total drive capacitance as a function of displacement is $C_{d}(t)=C_{d}(x(t))=\frac{\varepsilon A}{g-x(t)}=C_{d n} * \frac{1}{1-\chi(t)}$, where $C_{d n}=\varepsilon A / g$ is nominal drive capacitance, and $\chi(t)=x(t) / g$ is gap normalized displacement. Taylor series expansion method for nonlinear parallel plate capacitance as a function of the resonator's displacement yields $C_{d}(t)=C_{d n} \frac{1}{1-\chi(t)}=C_{s n} \sum_{n=0}^{\infty} \chi^{n}(t)$. 


$$
\text { Finally, } \quad \frac{\partial C_{d}}{\partial x}=C_{d n} \frac{\partial}{\partial x}\left(\frac{1}{1-\chi(t)}\right)=\frac{C_{d n}}{g} \frac{1}{(1-\chi(t))^{2}}=\frac{C_{d n}}{g} \sum_{n=0}^{\infty}(n+1) \chi^{n}(t) \text { and the full equation of }
$$

resonator's dynamics in terms of normalized displacement $\chi(t)$ can be written as

$$
\ddot{\chi}(t)+\frac{\omega_{n}}{Q} \dot{\chi}(t)+\omega_{n}{ }^{2} \chi(t)=\frac{C_{d n}}{2 m g^{2}}\left\{\left(V_{d c}^{2}+\frac{1}{2} v_{a c}^{2}\right)+\left(2 V_{d c} v_{a c} \sin \left(\omega_{d} t\right)\right)-\left(\frac{1}{2} v_{a c}^{2} \cos \left(2 \omega_{d} t\right)\right)\right\} \sum_{n=0}^{\infty}(n+1) \chi^{n}(t) .
$$

Derived equation has an infinite power series of $\chi(t)$ on the right hand side. The zero order approximation is obtained by neglecting linear and higher order terms on the right hand side. The resulting equation is identical to the full equation of motion for the case of displacement-linear lateral comb drive capacitor. It implies resonance of the structure in two cases: providing $\mathrm{AC}$ voltage at resonance frequency or at its half.

A more adequate approximation of parallel plate driven dynamics is given by linearization of the right hand side with respect to gap normalized displacement $\chi(t)$. The resulting equation approximates the nonlinear dynamics with an LTV system, which can be further simplified if we assume $V_{d c}>>v_{d}$. Finally, we get a system described by non-homogeneous Mathieu equation with harmonic inputs on the right hand side. This approximation of original nonlinear dynamics is still very coarse because nonlinear capacitive terms are not included. We experimentally demonstrated that these nonlinear terms become critically significant when motional amplitude is comparable to capacitive gap. However, the simplified LTV equation allows predicting phenomena of parametric resonance. Particularly, we can expect the system to develop significant amplitudes of motion when driving $\mathrm{AC}$ voltage is applied at double of resonant frequency (taking into account frequency down-tuning due to DC voltage). The motion occurs at the tuned resonant frequency, which is half of the driving $\mathrm{AC}$ voltage frequency. In the following section we experimentally characterize this nonlinear actuation method.

\subsection{Experimental Characterization of Nonlinear Parametric Excitation}

In this section we present our results and preliminary conclusions on experimental demonstration of the parametric double frequency actuation method using prototypes of distributed mass gyroscope fabricated in EFAB process.

The experimental setup consists of an EFAB distributed mass gyroscope prototype packaged in a DIP-40 ceramic package, which was installed on a breadboard with a trans-impedance amplifier. The tested mode of vibration is parallel plate actuated and sensed, with natural frequency $\sim 855 \mathrm{~Hz}$ and quality factor $Q=280$. The capacitive gap between 20 micrometers wide parallel plates is 30 micrometers. The DC bias voltage of $100 \mathrm{~V}$ was applied to the body of the resonator. Driving AC voltage was imposed onto anchored parallel plate structure. Sensing of the motion was done using anchored parallel plate structure, which was virtually grounded by means of trans-impedance amplifier with a $1 \mathrm{MOh}$ feedback resistor. The sense capacitor between fixed and moveable parallel plates has a fixed DC voltage difference across it. Velocity dependent motional current flows through the feedback resistor of the trans-impedance amplifier. Also, capacitive coupling between drive and sense electrode contributes to the output current. The pick-up current was converted to voltage and fed into a signal analyzer for spectral monitoring and data capturing.

During the experiment we control the driving AC voltage frequency wd, sweeping it in the vicinity of double of the natural frequency of the resonator (i.e. $w_{d} \sim 2 w_{n}$ ). The amount of motion was detected visually using a microscope (see Fig. 11). At the same time the FFT of the pickup signal was continuously performed, updated, and displayed by the dynamic signal analyzer (see Fig. 12). We compare results of FFT at resonance and when there was no visible amount motion (i.e. motional amplitudes were smaller than $\sim 0.5$ micrometer). From this comparison we determine the frequency content of the feed-through and distinct features of the signal in presence of motion.

We register significant amount of motion when the driving $\mathrm{AC}$ voltage was provided at frequency close to double of resonant frequency (i.e. $w_{d}=2 w_{n}=2 * 850 \mathrm{~Hz}=1.7 \mathrm{KHz}$ ). The amplitude of motion was approximately two times higher compared to the regular excitation of motion using the same combination of DC and AC voltage of resonant frequency. Due to capacitive parasitics coupling and non-ideality of the AC voltage generator, the feed-through of the drive occurs at all harmonic frequencies (i.e. $n^{*} w_{n}=\{0.85,1.7,2.55,3.4,4.25,5.1, \ldots\} \mathrm{KHz}$ ), which was equivalent to all even order harmonics of resonant frequency wd. However, the motion occurs at the resonant frequency (which is sub-harmonic of the drive $\mathrm{AC}$ voltage frequency) and all of its harmonics (i.e. $\left.n^{*} w_{n}=\{0.85,1.7,2.55,3.4,4.25,5.1, \ldots\} \mathrm{KHz}\right)$. In the 
pick-up signal odd order harmonics of resonant frequency represent purely the motion, (frequencies $(2 \mathrm{n}+1) w_{n}=\{0.85$, $2.55,4.25, \ldots\} \mathrm{KHz})$. Even order harmonics of resonant frequency contain both motional and parasitics signals.

\subsection{Preliminary conclusions on nonlinear parametric excitations}

Nonlinear parametric excitation of motion at resonant frequency by a super-harmonic voltage was demonstrated on prototypes of distributed mass gyroscopes. This non-LTI actuation approach experimentally exhibits several important advantages over conventional driving schemes. These include:

- high gain

- motional amplitudes as high as $80 \%$ of the capacitive gap

- $\quad$ strong frequency separation between motion and $\mathrm{AC}$ voltage feed-through

Distributed mass gyroscope is designed to have wide bandwidth drive mode without sacrificing the drive gain. Parametric double frequency drive scheme can potentially be used to provide distributed mass gyroscope with even wider bandwidth and higher gain by combining Coriolis responses from parametrically excited drive mode oscillators. However, detailed analytical and numerical study of the nonlinear dynamics associated with this driving scheme is needed in order to formulate functional control laws.

\section{ACKNOWLEDGEMENTS}

This research is supported by the National Science Foundation Grant CMS-0409923. Also we would like to acknowledge the following organizations and individuals: Integrated Nano-Research Facility (INRF) at University of California, Irvine for help with SCS-SOI fabrication; Microfabrica for fabricating EFAB prototypes; Max Perez and other members of UCI Microsystems lab for technical discussions.

\section{REFERENCES}

1. C. Acar, A. Shkel. Distributed-Mass Micromachined Gyroscopes for Enhanced Mode-Decoupling. IEEE Sensors Conference, September 2003, Toronto, Canada.

2. C. Acar, A. Shkel. Enhancement of Drive-Mode Bandwidth in MEMS Vibratory Gyroscopes Utilizing Multiple Oscillators. Solid-State Sensor and Actuator Workshop, Hilton Head Island, SC, June 2004.

3. C. Acar, and A. Shkel. An Approach for Increasing Drive-Mode Bandwidth of MEMS Vibratory Gyroscopes. Journal of Microelectromechanical Systems, Vol. 14, No. 3, June 2005

4. M. Handtmann, R. Aigner, A. Meckes, and G.K.M. Wachutka. A Sensitivity Enhancement of MEMS Inertial Sensors Using Negative Springs and Active Control. Sensors and Actuators A, Vol. 97, 2002, pp. 153-160. 


\section{ILLUSTRATIONS}

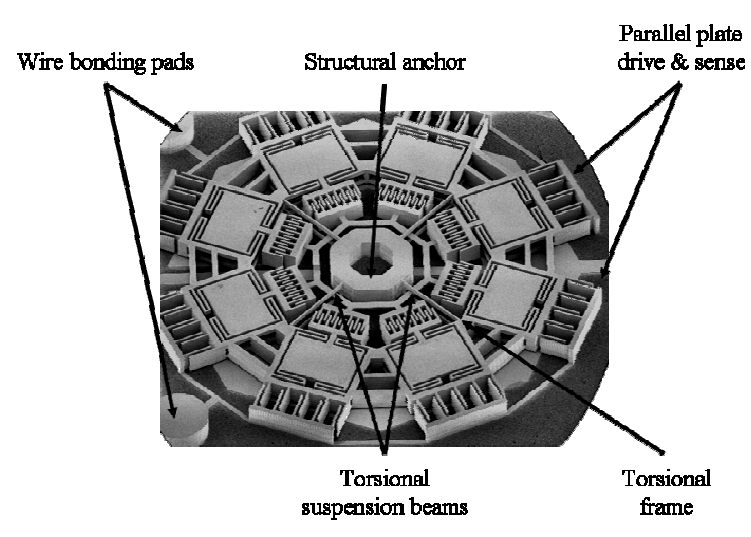

Figure 1. Scheme of the distributed mass gyroscope, fabricated in EFAB process.

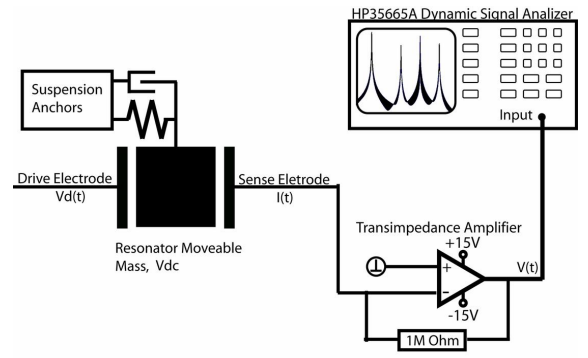

Figure 2. Electrostatic characterization of gyroscope's vibrational mode. Due to stray electrical coupling between anchored drive and sense electrodes the output signal also contains feed-through of the driving voltages.

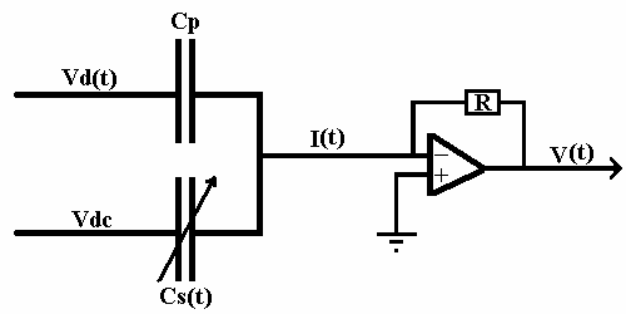

Figure 3. Electrical model of capacitive parasitics circuit in the gyroscope's drive mode resonator. The pick-up current is a sum of motional current, caused by variations in sense capacitor $\mathrm{Cs}(\mathrm{t})$ and feed-through current, caused by parasitic coupling between sense and drive electrodes.

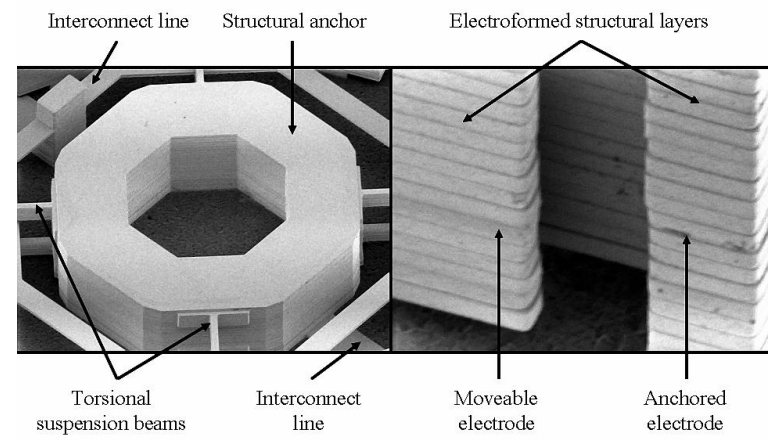

Figure 4. SEM micrograph gallery of EFAB prototypes shows 3D structures, consisting of multiple metallic layers. 


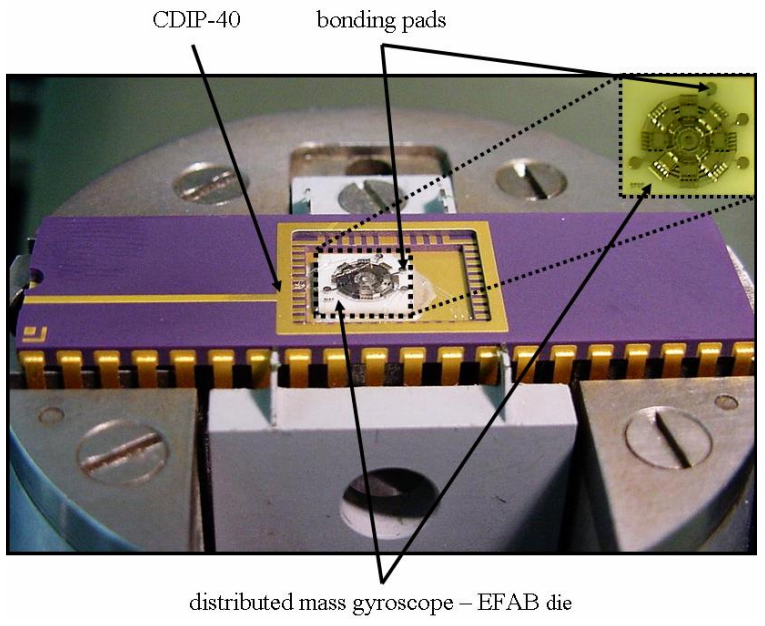

Figure 5. Photograph of distributed mass gyroscope die fabricated in EFAB process, packaged in DIP-40 ceramic package, and wirebonded.

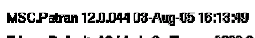

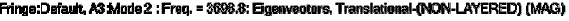

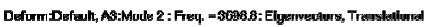
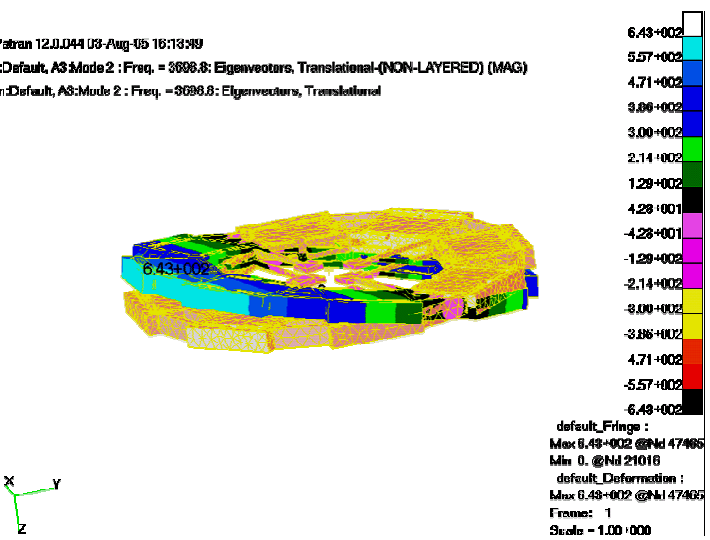

Frame: 1 ,

Figure 6. FEA results confirm that undesired out-of-plane modes have frequencies several times higher than main modes.

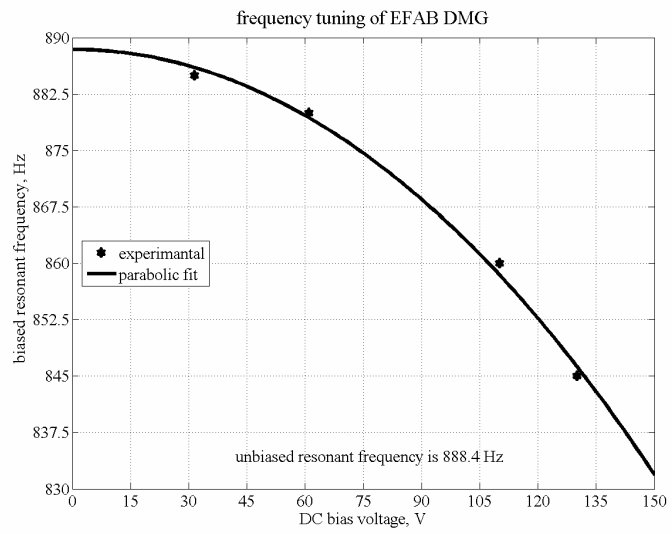

Figure 7. Parallel plate frequency tuning for EFAB distributed mass gyroscope. The linear negative electrostatic spring model fit allows identifying unbiased and biased values of resonant frequency.

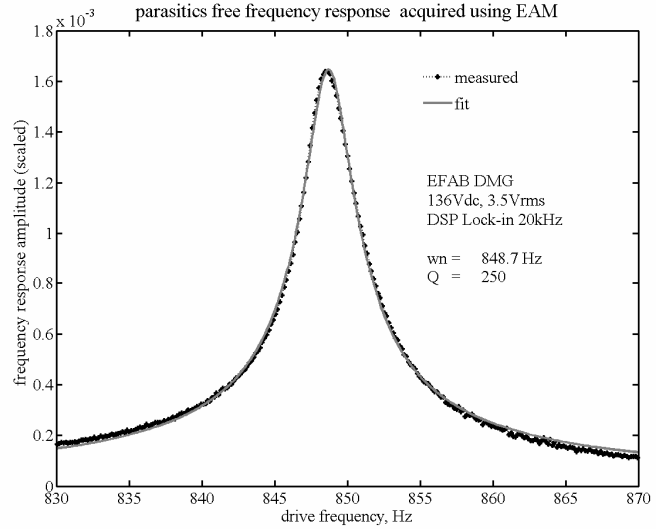

Figure 8. Parasitics free frequency response acquired using EAM technique. The analytical fit yields values for tuned resonant frequency $(850 \mathrm{~Hz})$ and atmospheric pressure Q-factor (250). 

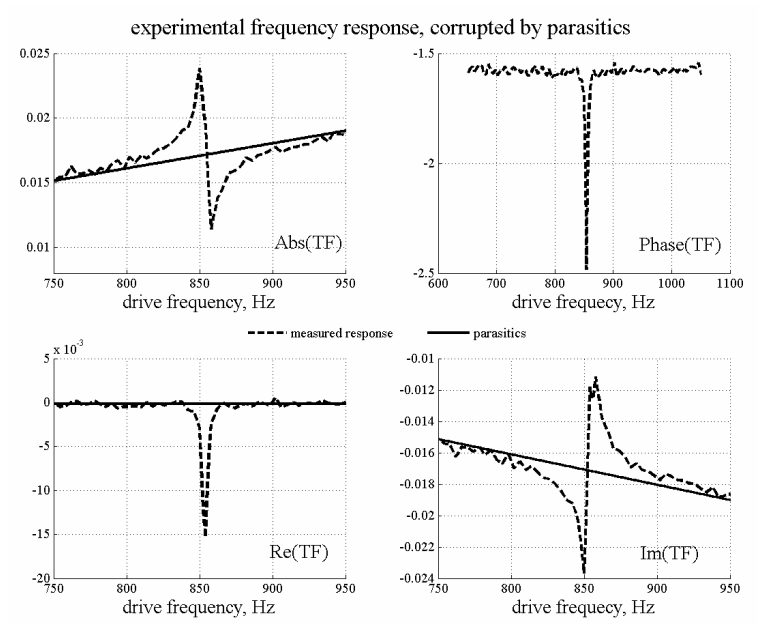

Figure 9. Experimental frequency response with parasitics. Parameters of parasitics model can be identified from analyzing real and imaginary parts separately. For EFAB process parasitics is identified to be purely capacitive, being represented by a linear trend in the imaginary part of experimental transfer function.

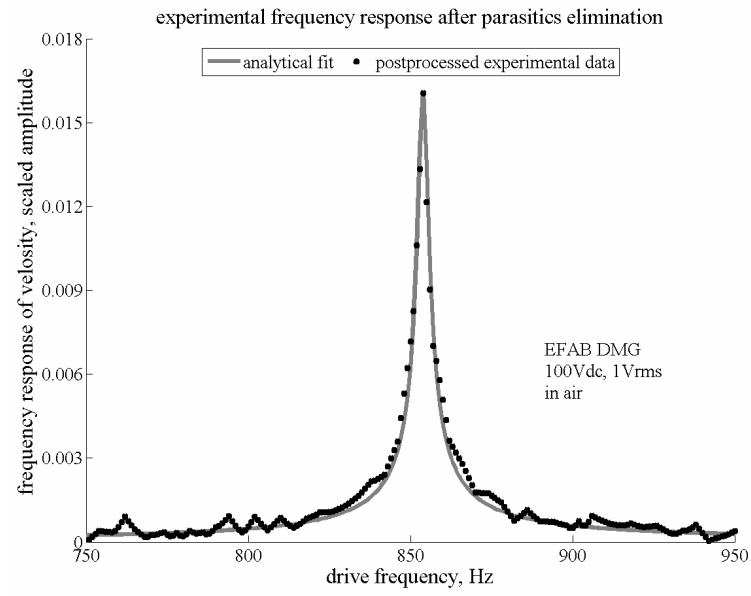

Figure 10. Experimental response after parasitics elimination. Analytical fit gives values for resonant frequency $(854 \mathrm{~Hz})$ and atmospheric pressure $Q$-factor $(200-250)$

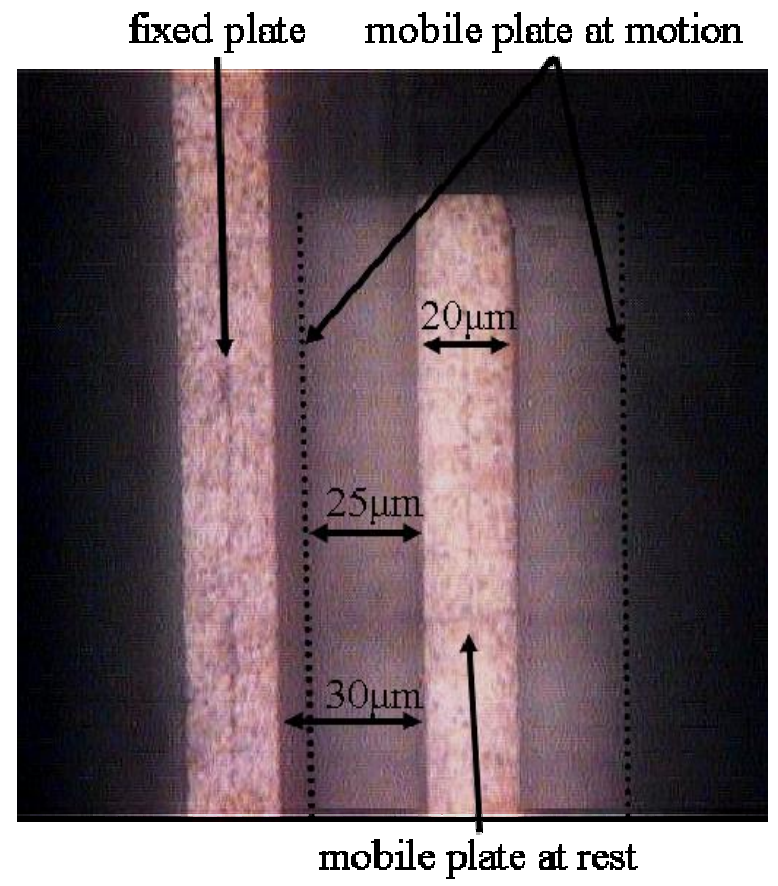

Figure 11. Optical photograph of resonant motion excited by a super-harmonic AC voltage. Identical pictures were taken at rest and at nonlinear parametric resonance and imposed on each other. The blurred area shows amplitude of motion ( $\sim 80 \%$ of the gap).

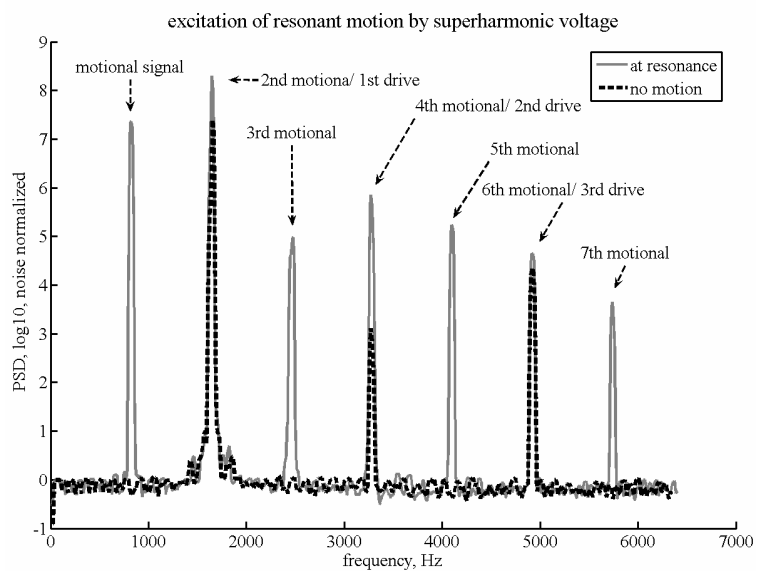

Figure 12. Sense signal PSD for parametrically excited gyroscope prototype. The driving AC voltage is provided at double of resonant frequency. The strong frequency separation between first (main) motional component and parasitics is achieved. 